\title{
Results S1
}

\section{Results S1. Isolation of potato GA 3-oxidase clones}

Using degenerated oligonucleotides ( $\mathrm{H} 5$ and $\mathrm{H} 7)$ a fragment of the expected molecular weight (460 bp), named B3ox, was amplified, cloned and sequenced, sharing $57 \%$ identity at the amino acid level with the protein At3ox2. The B3ox fragment exhibited some of the conserved regions present in all plant dioxygenases as well as certain motifs specific to the GA3ox genes (Figure S1). Furthermore, B3ox hybridized to a messenger exhibiting negative feed-back regulation by $\mathrm{GA}_{3}$ (Figure $\mathrm{S} 1$ ), suggesting that this partial clone corresponds to a GA 3-oxidase-encoding transcript. Using the B3ox fragment as a probe, a cDNA library from leaves of the potato ga1 mutant (Carrera et al., 1999) was screened. We obtained several clones (Figure S2A) that were copies of the same gene, because they shared identical nucleotide sequences in their overlapping regions. In addition, two other full-length cDNAs were isolated which included an unspliced intron in the protein coding region. The presence of this intron resulted in a shift in the protein reading frame, allowing for a truncated protein that lacked any catalytic activity. Unspliced StGA3ox2 RNAs seemed to be relatively abundant as several of the isolated clones contained such intron. 